\title{
Application of Computer-Aided to Improve Industrial Productivity in Cement Factories by using a Novel Design of Quantitative Conveyor
}

\author{
Anh Son Tran ${ }^{1}$ \\ Dept. of Manufacturing Engineering \\ HCMC University of Technology, VNU-HCM \\ Ho Chi Minh City, Vietnam
}

\author{
Ha Quang Thinh Ngo ${ }^{* 2}$ \\ Dept. of Mechatronics Engineering \\ HCMC University of Technology, VNU-HCM \\ Ho Chi Minh City, Vietnam
}

\begin{abstract}
To deal with the industrial evolution 4.0, many cement enterprises must be enhanced the productive ability. In this paper, the novel design of quantitative conveyor is introduced to improve the industrial productivity. Firstly, the test hardware platform is set-up based on customer's requirement. Then, the analysis and control of mechanical platform for quantitative conveyor is investigated. From the experimental results, the operation of conveyor is stable and precise to ensure the output products for cement factories.
\end{abstract}

Keywords-Motion control; cement industry; conveyor; automation; robotics system

\section{INTRODUCTION}

The more the economic develop, the more building is constructed. Many essential components of constructions such as cement; steel or concrete play an important role. For producers of cement, it is important to visualize the amount of this material. By knowing this information, it is needed for business trade, storage and distribution management. Since the need of cement becomes very large in market, its amount per day is huge and continuously. Hence, it is most often measured increment. In other words, it measures the volume of transported material. Traditionally, workers must measure the weight of cement in manual labor or in separated unit. This job is very limited because it costs much and low productivity. Furthermore, it is difficult to maintain the precise measurement due to restricted human resource. In reality, the weight is measured during the transport by means of conveyor. The problem is that the complicated measuring process and calibrating method often brings troubles to operators.

In the other fields, for example wood chips or mining, it is also required to maintain the industrial production and enhance the production line. In $[1,2]$, researchers introduced a mathematical relationship of computing volume measuring by a laser scanning method during transport on conveyor. The scanner SICK LMS400 hanging above conveyor and industrial PC monitoring overall process are combined together. The belt speed is constant and its information is transmitted via digital line. However, the cost of this method is expensive because of using laser scanner, it is hard for maintenance job. Especially, it cannot estimate the wood humidity. The study [3, 4] presented a distributed model of the transport conveyor which material moves along at the same speed to obtain the equations in closed form. Based on this model, the time delay between the arrival of the material at the inlet of the conveyor line and its outlet from the conveyor is calculated. Actually, the distribution of material on conveyor is not regular. And the material must be granulated form.

To control and monitor the conveyor system, a centralized system [5-8] including S7-300 PLC and peripheral devices is implemented. This system is to transport coal mine using PLC and touch screen to visualize. The hardware and software are matched together via Profibus DP. The components of this system are popular in market and un-expensive. Though, the effectiveness and feasibility of proposed system should be considered since this paper is just simulated. Once, these works in $[9,10]$ suggested a simulation tool for belt conveyor in bulk material transportation. They concentrate on role of conveyor in contribution to heavy industry. The results of research help the design process more rapid and simplified. The developers could shorten consuming time by analyzing the simulative data. The more convenient transportations are to apply the autonomous system in workshop [11-13]. In this method, a number of vehicles continuously operate to carry cargo around the workplace. Nonetheless, these drawbacks such as light weight carriage, sudden accidence or high maintenance cost could impact on the overall effectiveness of whole system.

In this paper, the overall scheme of quantitative conveyor for cement industry is proposed. From practical acquirements, the hardware platform is built up in the first period. Later, the system software and control algorithm are described in detail. Several experiences in calibrating method are discussed since proposed platform is operated. Finally, some conclusions are carried out for future development.

\section{BASIC PRINCIPLE OF OPERATION}

There are several ways to measure how weight material is on conveyor. However, the effective and feasible method are not interested so much. As a result, the description of design system is detailed in Fig. 1. After material is poured, the belt conveyor brings matter based on rollers. The load-cell is located on one of rollers. This component is considered as weightbridge. The area in front of and after load-cell, named weight span, has length L. The controller collects signals from load-cell and speed sensor to calculate system parameters such as rate, load.

*Corresponding Author. 


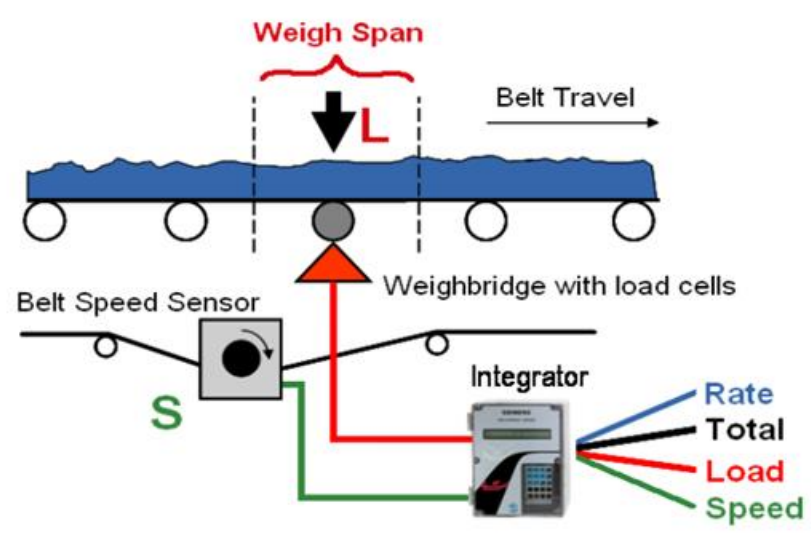

Fig. 1. Illustration of Proposed Approach.

In market, the products derived from cheal level are often large error, low firmness and suitable for small business. The others are expensive, high reliability and proper for mass production. For developing countries, there is a need to

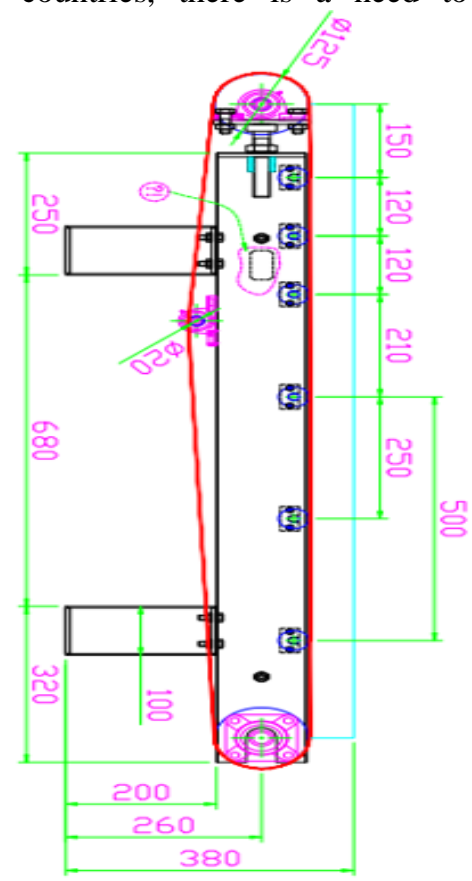

(a) introduce a conveyor system which is high quality, low cost and durable. To solve the problems, the design process should be optimized and control method need to be effective and applicable.

\section{MEChanical Model OF CONVEYOR SySteM}

In this section, a belt conveyor with medium size is studied. The components platform as Fig. 2 are described in transportation system labeling as following. From feeding funnel, material drops on belt body and is delivered regularly by lever. There are many rollers distributed evenly along the length of conveyor. The rollers classifying into two sub categories, passive roller and active roller, are placed under belt. The active one is connected to driving motor directly while the others keep belt on a plane. To prevent the dimple, the adjusting bolt to tense belt is located in one side. The operator could handle to enhance the accuracy measurement. The material passing the area of weight span is estimated through load cell. The data is feedback to controller in real time to synthesize. The industrial productivity is able to visualize continuously.

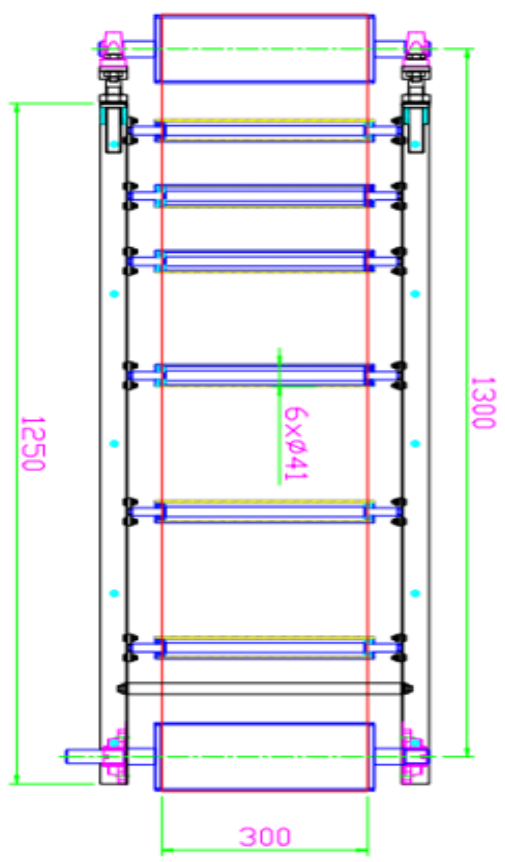

(b)

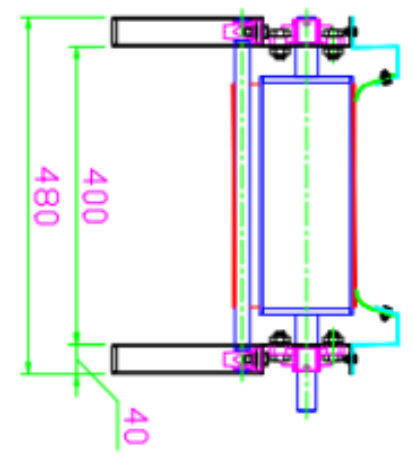

(c)

Fig. 2. Mechanical Scheme of Conveyor System, (a) Side View, (b) Upper View and (c) Front View. 


\section{MONITORING METHOD AND CONTROL APPROACH}

The monitoring and control of dynamic quantitative conveyor is based on integrated model, transpoting and weighing, when it operates. For easy supervision, HMI touch screen helps to show system parameters and control status. The controller obligates to certify system reliability, high repeated precision and standardization. The power circuit is embedded into inverter to drive AC motor. The data fusion between load cell and speedometer gives exact information to main CPU. In fact, there are two options instead of using load cell. Magnetoelastic sensor, being truly non-contact based, have inherent advantages over traditional solutions based on strain gauges regarding robustness and cost structures. A piezoelectric sensor is a device that uses the piezoelectric effect, to measure changes in pressure, acceleration, temperature, strain, or force by converting them to an electrical charge. Nevertheless, torque or load sensor will play an increasingly important role in many applications, enable critical benefits such as efficiency improvements, weight saving, operational safety, enhanced productivity and cost saving.

Since load sensor returns analog signal to controller while main CPU serves digital pulse as Fig. 3. A high precision analog-digital converter is necessary to add in control scheme. The more accuracy the converter is, the more exact the measuring data is. Additionally, the processing measurement results holds an important role. Each load sensor requires the particular processing algorithm. If the algorithm converges faster, the consuming time is shorter. The concerning system coefficients are length of weigh span, sampling time and velocity of conveyor.

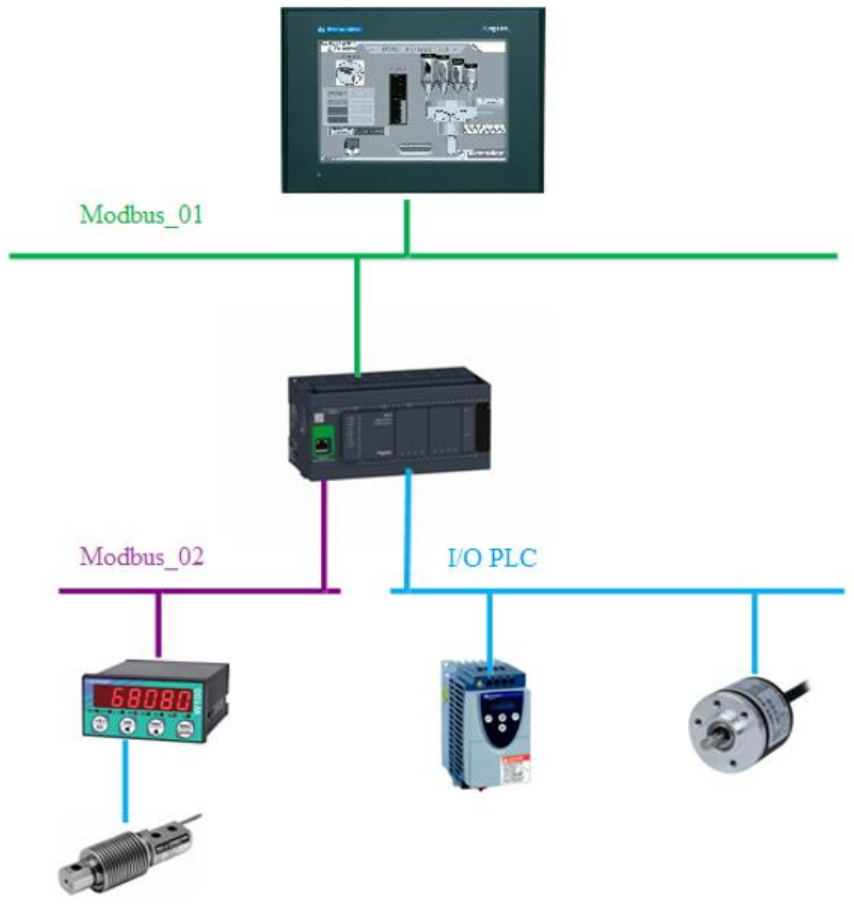

Fig. 3. Overview of Distributed Control Diagram.

\section{DESIGN OF Industrial CONTROLlER}

The embedded controller plays an important role in overall system. To interact with technical engineer, it is necessary to design a graphical tool under computer-aided. This program would handle entire missions in system: manage control signal and coefficients, control dynamic motor, output command values from controller and so on. In Fig. 4, the computer-aided software to design an interface on touch screen monitor is shown. Owing to its, the designer could carry out friendly program, fast debugging and easy to maintenance. After various tests and calibrations, a release version of graphical database has been packaged in Fig. 5.

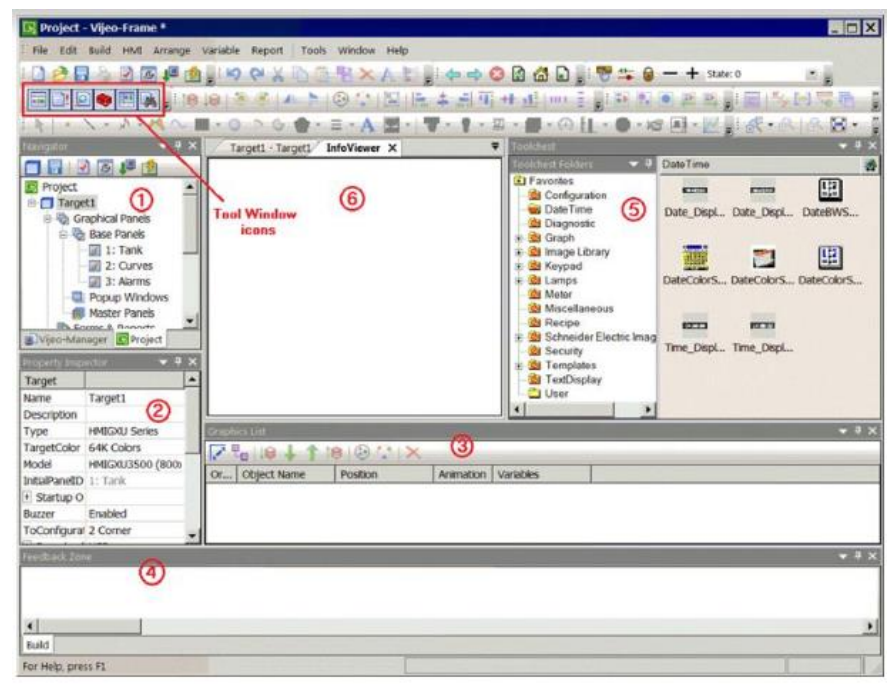

Fig. 4. Computer-Aided Graphical user Interface Program.

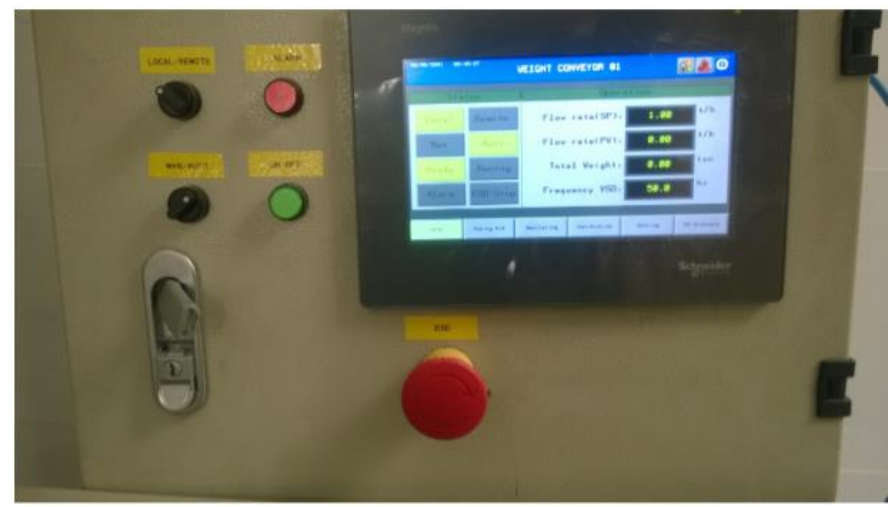

Fig. 5. Control Program of Embedded Controller on Central Panel.

\section{RESULTS AND DISCUSSION}

To verify the proposed design, the hardware platform is completed as Fig. 6. In this paper, a belt conveyor with medium size which is popular in developing country is studied. From this experimental model, a large-scale conveyor system could be considered to develop in future. After setting up the system instruments, the data connection is established. The control panel could be placed far from system, for instance in central control room, for convenient work. Fig. 7 shows practical layout of electrical control box. For the electrical safety, main CPU and AC inverter are separated if emergency case occurs. All electrical contact points are covered by 
isolation plastic elements. The outside layout of control panel box has monitoring screen, five buttons such as emergency stop, on, off and local/remote. LED signals perform status machine visibly.

Most of devices are from Schneider company except incremental encoder from Autonics Ltd. company and indicator from Laumas firm. The communication protocol is chosen as modbus network since the ability to reject noise of measurement system. All data wires are covered by shield outside.

The graphical user interface leaning on SCADA is demonstrated in Fig. 8. The AC inverter ATV11 drives feeding conveyor system depending on setting values from SCADA. PLC module is connected with inverter by analog signal, communicates with weight indicator W100 via Modbus protocol.

In Fig. 9, a monitoring page of control parameters is displayed to follow control process. The options Pid/Limit/Overflow indicate status of PID function. To tune PID coefficients, Man/Reset button is pressed to adjust up or down value. The chart on right side reveals information of SP/PV/Limit or flow. Based on experience of operator, it can be seen that the proposed system works well in industrial environment.

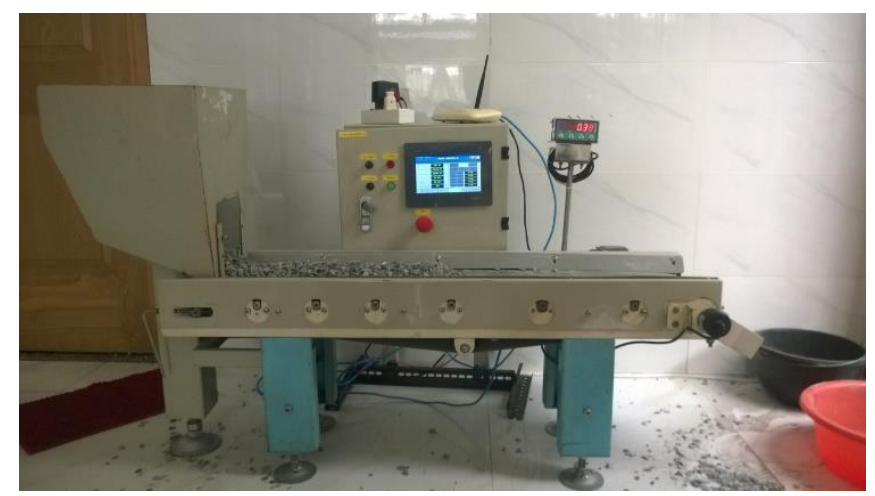

Fig. 6. Experimental Model of Proposed Material Transportation System.

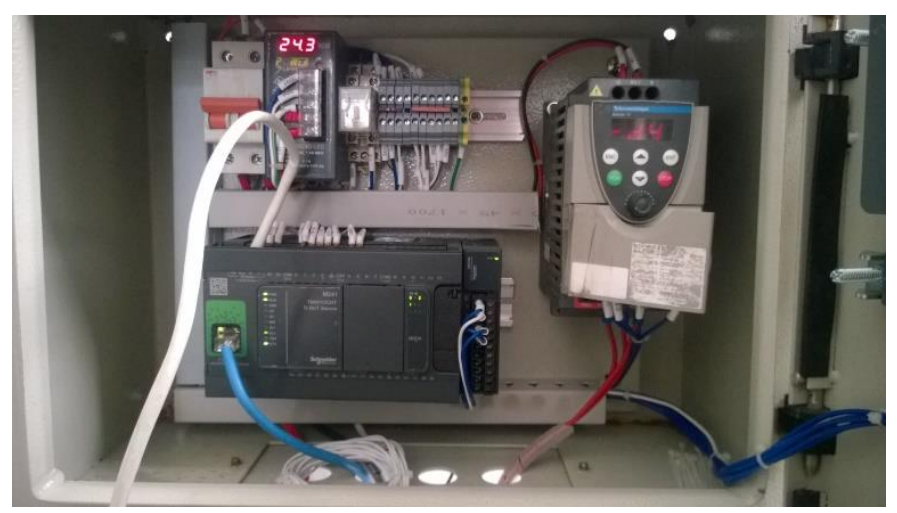

Fig. 7. Layout Inside Electrical Control Box.

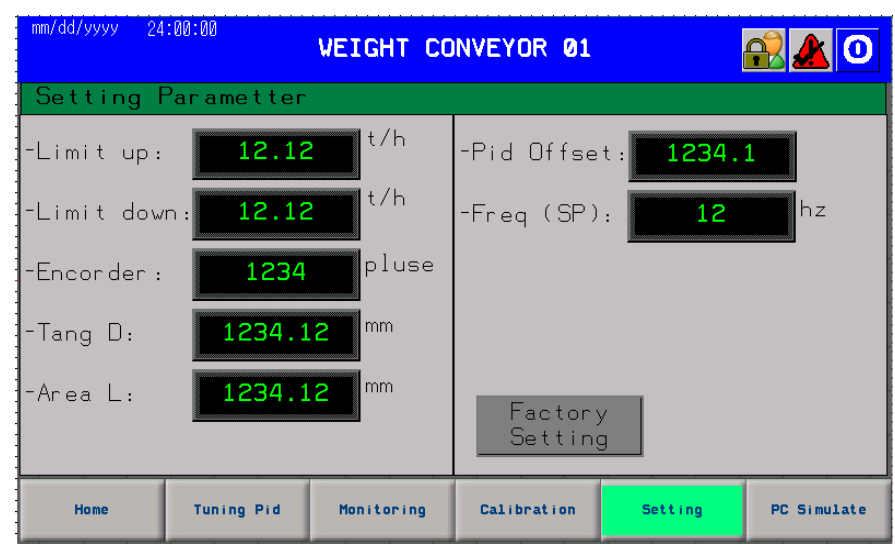

Fig. 8. Experimental Software of Proposed Material Transportation System.

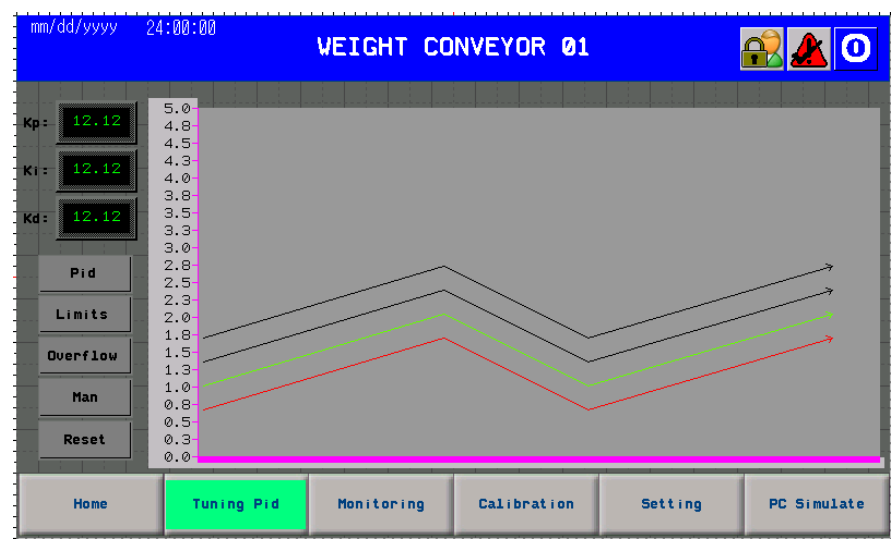

Fig. 9. Experimental Result of Tuning PID Control.

\section{CONCLUSIONS}

In this paper, a medium sizing material transportation system is researched. From practical demand of industry, the conveyor system that guarantee high productivity and enhance precise measurement as well, is developed. The mechanical design and method to weight are considered to optimize the initial cost, easy to maintain and high production. From experimental results, it has been proved that the proposed system is effective and feasible in reality. It could be applied in various fields such as mining, cement.

\section{ACKNOWLEDGMENT}

The authors would like to thank the helping of Can Tho cement factory. This work is funded by Ngo Ha Gia Ltd. Co. under research grant no. NHG19-012-0008.

\section{REFERENCES}

[1] D. Fojtik, "Measuring of the volume of material on the conveyor belt," Proc. of the 2014 International Carpathian Control Conference, pp. 121$124,2014$.

[2] H. Q. T. Ngo, T. P. Nguyen, T. S. Le, V. N. S. Huynh and H. A. M. Tran, "Experimental design of PC-based servo system," IEEE International Conference on System Science and Engineering, pp. 760$765,2017$. 
[3] O. Pihnastyi, V. Khodusov, "Model of a composite magistral conveyor line," IEEE First International Conference on System Analysis \& Intelligent Computing, pp. 1-4, 2018.

[4] H. Q. T. Ngo, Q. C. Nguyen and T. P. Nguyen, "Design and implementation of high performance motion controller for 2-D delta robot," IEEE International Conference on Information Science and Technology, pp. 129-134, 2017.

[5] Q. Lu, X. Wang and L. Zhuang, "Research and design of monitoring system for belt conveyor," International Conference on Computer Science and Service System, pp. 1943-1945, 2012.

[6] H. Q. T. Ngo and A. S. Tran, "Using Fuzzy Logic Scheme for Automated Guided Vehicle to Track Following Path Under Various Load," IEEE International Conference on Green Technology and Sustainable Development, pp. 312-316, 2018.

[7] Y. G, K. Hu, P. Wang and Y. Wang, "Dynamic simulation of belt conveyor based on virtual prototyping," International Conference on Mechanic Automation and Control Engineering, pp. 5986-5988, 2010.

[8] H. A. M. Tran, H. Q. T. Ngo, T. P. Nguyen and H. Nguyen, "Implementation of vision-based autonomous moblie platform to control $\mathrm{A}^{*}$ algorithm," IEEE International Conference on Recent Advances in
Signal Processing, Telecommunication and Computing, pp. 39-44, 2018.

[9] H. Q. T. Ngo, M. H. Phan, "Deisgn of an Open Platform for MultiDisciplinary Approach in Project-Based Learning of an EPICS Class," Electronics, 8(2), 200, 2019.

[10] L. Guangbu, L. Ruqiong, "Belt conveyor modeling and performance simulation based on AMESim," International Conference on Information and Computing Science, vol. 4, pp. 304-307, 2009.

[11] H. Q. T. Ngo, T. P. Nguyen and H. Nguyen, "The fusing framework between lifting carrier and tractor-trailer for modern transportation," Advances in Science, Technology and Engineering System Journal, vol. 4, no. 4, pp. 522-528, 2019.

[12] H. Q. T. Ngo, T. P. Nguyen, H. Nguyen, "A complete comparison to design complementary filter and kalman filter for aerial vehicle," International Journal of Mechanical Engineering and Technology, vol. 9, pp. 502-513, 2018

[13] Q. T. Truong, H. Q. T. Ngo, T. P. Nguyen, H. Nguyen and W. H. Kim, "A novel infrastructure design of industrial autonomous system," International Journal of Fuzzy Logic and Intelligent Systems, vol. 19, no. 2, pp. 103-111, 2019. 